Research

\section{Investigate, study, explore, examine, enquire, seek}

\section{MacAuley}

\section{Research is essential for the development of sports medicine as a specialty}

W

e need to invent a new word. A word with the same meaning but without the baggage. Few would argue with the principle that, as a medical discipline, we should examine current practice to see if it is effective, and constantly search for new treatments, therapies, and techniques. But mention research in sports medicine and see your friends and colleagues melt away. Let's demystify this word: research is simply asking a question about what we do in a structured way.

You are a scientist. You may have forgotten now, but you started off in a scientific discipline asking research questions. As clinicians, the first step is to think about what we do: to wonder if what we do actually works. We need help from someone who can nurture our ideas in these early stages and refine these questions. Just as you would use the techniques and skills of any sport, there are skills needed in research. We can learn from other disciplines, and sports science research, in particular, has developed much faster than clinical sports medicine. Perhaps because it is laboratory based, it may be easier to take the early steps. But we should learn from our sports science colleagues. They have knowledge and expertise and a track record in research. Few sports medicine doctors have expert knowledge of epidemiology or statistics, the building blocks of research. Questionnaire design, choosing a population, selecting a sample are just a few of the key areas in structuring and research study. We need to learn to seek help early and often, particularly in the planning of any research study. A mistake in the method can never be retrieved.

"... and promote the greatly underdeveloped research dimension of sport and exercise medicine"

Research is essential for our development as a specialty. We must be able to compare ourselves with other specialties and show evidence of our maturity as a discipline. We can ably show evidence of two of the three main traits of a medical specialty: our own body of clinical skills and validated high quality training schemes. But where is the body of research supporting our clinical discipline? Masters degrees are our research nursery. Anyone who has registered for a masters degree in sports medicine will be expected to submit a project. This might be a simple piece of research, a literature review, or an audit. Doing a masters degree is a learning process. The outcome is that you know how to do a piece of research correctly. On completion, you are now one of the few experts in sports medicine research and have some responsibility to help teach the sion is from a simple project to an MSc thesis and ultimately a PhD. Not all of us need make the full transition. But some should. To develop a critical mass of researchers, we must build the pyramid from the base, create networks, groups of people working together, and promote the greatly underdeveloped research dimension of sport and exercise medicine. next generation. The natural progres-
Few ever forget presenting their first poster, giving a presentation at a conference, or seeing their name in print for the first time. Even seasoned researchers feel a frisson of excitement when they see their work in print- that someone should value their piece of work so much that it is worthy of publication. If a piece of work is worth doing, it is worth sharing and we all learn from each others work. Those conferences that publish abstracts allow researchers to get their foot on the first rung of the publication ladder. Our annual BASEM conference should be an opportunity to learn how to present new work among friends.

Focus on goals, not barriers. That is what we tell our athletes. There are obstacles and difficulties in undertaking research too. Athletes know about preparation, planning, targets, and goals. They know that every performance needs hours, weeks, months, and even years of training, overcoming barriers and setbacks along the way. Planning a major research project is similar. And, like athletic performance, we need technical help from experts, advice from the experienced, and support when things wrong. Funding, ethical permission, and recruiting patients are the major hurdles. Research ethics committees can be frustrating. But remember they are there to protect patients, you and me, from inappropriate research and researchers. Planning research projects is like planning preparation for a sporting event. Ask for help first. You wouldn't set off to sail around the world, learn hang gliding, or rock climbing without expert advice. Similarly, seek expert guidance in research. Doing good research well requires thought, planning, preparation, and effort. But the key ingredient is in finding people with ideas and enthusiasm. No different from sport. And it can be almost as much fun too.

Br J Sports Med 2005;39:64. doi: 10.1136/bjsm.2004.016493

Correspondence to: Professor MacAuley, University of Ulster, Belfast, Northern Ireland, UK; domhnall.macauley@ntlworld.com

Competing interests: none declared 